\title{
Molecular and epidemiological characterization of bovine intrauterine Escherichia coli
}

\author{
R. C. Bicalho, ${ }^{1}$ V. S. Machado, M. L. S. Bicalho, R. O. Gilbert, A. G. V. Teixeira, L. S. Caixeta, \\ and R. V. V. Pereira \\ Department of Population Medicine and Diagnostic Sciences, College of Veterinary Medicine, Cornell University, Ithaca, NY 14853
}

\begin{abstract}
Escherichia coli are believed to be associated with postpartum metritis and endometritis but their role in the pathogenesis of both diseases is still undefined. In this study, uterine swabs for E. coli isolation were collected from 374 lactating Holstein cows housed on 4 commercial farms near Ithaca, New York. A total, 125 of 374 cows $(33.4 \%)$ were positive for $E$. coli culture. Standard multiplex PCR protocols were used to screen the isolates for the presence of 32 virulence factor genes. Cows that had twin parturition were 4.4 times more likely to have intrauterine $E$. coli contamination than those that gave birth to single live female calves. Stillborn parturition and birth of single live male calves also increased the odds of intrauterine contamination by E. coli (3.7- and 1.6-fold, respectively) compared with birth of live female calves. Six virulence factors, common to extraintestinal and enteroaggregative $E$. coli, were found to be associated with metritis and endometritis: fim $H, h l y A, c d t, k p s M I I, i b e A$, and ast $A$. The virulence factor gene $\mathrm{fimH}$ was the most prevalent and the most significant: intrauterine $E$. coli carrying fimH and at least 1 of the other 5 identified virulence factors were pathogenic, and phylogenetic analysis based on the nucleotide sequence of DNA gyrase from 41 such IUEC revealed 2 clades.
\end{abstract}

Key words: dairy cow, Escherichia coli, endometritis, metritis

\section{INTRODUCTION}

Postpartum uterine diseases - specifically puerperal metritis, clinical endometritis, and subclinical endometritis - are important for animal welfare reasons because they contribute to cow discomfort and elimination from the herd and for economic reasons because they profoundly affect reproductive performance. Puerperal metritis is characterized by an abnormally enlarged uterus and a fetid, watery, red-brown uterine discharge,

Received June 21, 2010.

Accepted August 30, 2010.

${ }^{1}$ Corresponding author: rcb28@cornell.edu associated with signs of systemic illness (decreased milk yield, dullness, or other signs of toxemia) and fever $>39.5{ }^{\circ} \mathrm{C}$ within $21 \mathrm{~d}$ after parturition (Sheldon et al., 2006). Clinical endometritis is the inflammation of the endometrium and is characterized by the presence of purulent uterine discharge detectable in the vagina 21 d or more after parturition, or mucopurulent discharge detectable in the vagina after $26 \mathrm{~d}$ postpartum (Sheldon et al., 2006).

Escherichia coli are normal inhabitants of the gastrointestinal tract and play a pivotal role in mammalian health as a commensal organism. However, several strains of E. coli are capable of causing diseases in humans and animals, and some are extremely pathogenic such as the E. coli O157:H7 (Kaper et al., 2004). The role of E. coli in the pathogenesis of metritis and endometritis is poorly understood (Silva et al., 2009). Recently, 2 research teams have partially characterized different collections of $E$. coli isolates from the uterus of postpartum dairy cows (Silva et al., 2009; Sheldon et al., 2010). Silva et al. (2009) evaluated 15 E. coli virulence factor $(\mathbf{V F})$ genes and none were associated with uterine disease. A different study by Sheldon et al. (2010) evaluated $17 \mathrm{VF}$ genes and the VF fyuA was found to be associated with uterine disease; the expression of the VF gene fimH was also confirmed by an in vitro adhesion assay after mannose treatment. Hence, the role of E. coli in the pathogenesis of metritis and endometritis is still unanswered.

The objective of the present study was to evaluate the molecular epidemiology of $32 \mathrm{E}$. coli $\mathrm{VF}$ genes and their association with the incidence of postpartum metritis and endometritis using a large sample size of 374 lactating Holstein cows from 4 dairy farms.

\section{MATERIALS AND METHODS}

\section{Farms, Management, and Sample Collection}

Data were collected from 4 dairy farms located near Ithaca, New York, from August 17, 2009, to January 29, 2010. Farm A milked 2,800 cows, farm B milked 3,000 cows, farm C milked 1,600 cows, and farm D 
milked 1,000 cows. These farms were selected because of their long working relationship with the Ambulatory and Production Medicine Clinic at Cornell University (Ithaca, NY). The herds consisted of Holstein cows housed in freestall barns with wastepaper pulp bedding. Cows were milked 3 times daily in milking parlors. All lactating cows were offered a TMR consisting of approximately $55 \%$ forage (corn silage, haylage, and wheat straw) and $45 \%$ concentrate (corn meal, soybean meal, canola, cottonseed, and citrus pulp) on a DM basis of the diet. The diet was formulated to meet or exceed the NRC (2001) nutrient requirements for lactating Holstein cows weighing $650 \mathrm{~kg}$ and producing $45 \mathrm{~kg}$ of $3.5 \%$ FCM.

Uterine swabs were performed by the research team ( 5 veterinarians and 1 veterinarian student) on a total of 374 cows (200 cows in farm A, 70 in farm B, 63 in farm $\mathrm{C}$, and 41 in farm $\mathrm{D}$ ). The average DIM at sampling ranged from 2 to 7 . Uterine swabs were collected as follows: cows were restrained and the perineum area was cleansed and disinfected with a $70 \%$ ethyl alcohol solution. Then, a sterile swab (Double-Guarded Uterine Culture Swab, Har-Vet McCullough, Spring Valley, WI) covered by a sterile pipette (inside a plastic sheath) was introduced to the cranial vagina. The pipette was manipulated through the cervix into the uterus. There, the sheath was ruptured and the swab was exposed to uterine secretion. The swab was pulled inside the pipette and kept in transportation medium at $4^{\circ} \mathrm{C}$ until it was processed in the laboratory.

\section{Case Definition}

Metritis was defined as the presence of fetid, watery, red-brown uterine discharge, associated with systemic signs of illness and rectal temperature $>39.5^{\circ} \mathrm{C}$. A diagnosis of metritis was evaluated by the farm employees in the first 14 DIM. Clinical endometritis was evaluated in a subsample of cows that consisted of 117 cows; evaluation was performed by the research crew at $28 \pm$ 3 DIM. Clinical endometritis was defined as a presence of purulent or mucopurulent discharge, by retrieving vaginal mucus using the Metricheck device (Metricheck, SimcroTech, Hamilton, New Zealand) as described in a previous study (McDougall et al., 2007).

\section{Bacterial Isolation and DNA Extraction}

The uterine swabs were taken to the laboratory and cultured aerobically on MacConkey agar (Difco, BD Biosciences, Franklin Lakes, NJ) at $37^{\circ} \mathrm{C}$, and $E$. coli colonies were distinguished by a purple-red color. Five typical E. coli colonies from the MacConkey agar cultures were picked and subsequently streaked on
CHROMagar-E. coli (CHROMagar, Paris, France) for isolation and further identification of $E$. coli strains. Selected colonies were stored in Luria-Bertani (LB) broth containing $20 \%$ glycerol at $-80^{\circ} \mathrm{C}$, and DNA was extracted using InstaGene Matrix (Bio-Rad Laboratories, Hercules, CA) as described previously (Higgins et al., 2007).

\section{PCR and Gel Electrophoresis}

All reactions were performed in a $25-\mu \mathrm{L}$ volume using $24 \mu \mathrm{L}$ of $1 \times$ Green GoTaq Master Mix (made from $2 \times$ Green GoTaq Master Mix consisting of Green GoTaq Reaction Buffer, $400 \mu M$ dATP, $400 \mu M$ dGTP, 400 $\mu M \mathrm{dCTP}, 400 \mu M \mathrm{dTTP}$, and $3 \mathrm{mM} \mathrm{MgCl}{ }_{2}$; Promega Corp., Madison, WI) and primers, and $1 \mu \mathrm{L}$ of DNA extract. All thermal cycling protocols were performed in a 2720 Thermal Cycler (Applied Biosystems, Foster City, CA). Negative controls consisting of the PCR mixture without DNA were included in all PCR runs. Amplification products were separated by electrophoresis through a $1.2 \%$ (wt/vol) agarose gel, stained with 0.5 $\mu \mathrm{g} / \mathrm{mL}$ ethidium bromide, and visualized with a Kodak Gel Logic 100 Imaging System (GL 100, Scientific Imaging Systems, Eastman Kodak Co., New Haven, CT). Amplicons that had the expected molecular size were considered to be positives.

For further confirmation that the isolates belonged to the E. coli species, the 340-bp fragment of the bacterial $16 \mathrm{~S}$ rRNA-encoding gene was amplified by using the primers $16 \mathrm{SF}$ (5'-GTT AAT ACC TTT GCT CAT TGA-3') and 16SR (5'-ACC AGG GTA TCT AAT CCT GTT-3') as described previously (Malinen et al., 2003). All cultures that did not yield the 340-bp fragment in gel electrophoresis were excluded from the study. The genetic diversity of $E$. coli isolates was evaluated by randomly amplified polymorphic DNA (RAPD)-PCR with the informative primer 1283 (5'-GCG ATC CCC A-3') as described previously (Wang et al., 1993). The images were visually analyzed; isolates from the same cow (5 isolates per cow) were compared and E. coli colonies that presented distinct genome profiles were selected for subsequent analyses. After RAPD-PCR, $350 \mathrm{E}$. coli isolates, an average of 2.8 per positive cow, were selected for further analyses.

A multiplex $c d t$ PCR using 4 primer pairs was performed to detect $E$. coli isolates having sequences common to $c d t B$ genes, as described by (Toth et al., 2003). The thermal cycling conditions were $94^{\circ} \mathrm{C}$ for $5 \mathrm{~min}, 30$ cycles of $94^{\circ} \mathrm{C}$ for $1 \mathrm{~min}, 55^{\circ} \mathrm{C}$ for $1 \mathrm{~min}$, and $72^{\circ} \mathrm{C}$ for $1 \mathrm{~min}$, and a final step of $72^{\circ} \mathrm{C}$ for $10 \mathrm{~min}$ (Toth et al., 2003). Isolates that yielded a PCR product size of 466 bp were considered positive for the presence of $c d t B$ genes. 
A multiplex 16-plex PCR was conducted to assess $E$. coli isolates that presented genes related to diarrheagenic $E$. coli; 16 pairs of primers were used in this PCR to detect $16 \mathrm{VF}$ genes (eaeA, escV, ent, bfpB, EHEC-hly, stx1, stx2, ipaH, invE, astA, aggR, pic, elt, estIa, estIb, and uidA) as described (Antikainen et al., 2009). Cycling parameters were $98^{\circ} \mathrm{C}$ for 30 s, 30 cycles at $98^{\circ} \mathrm{C}$ for $10 \mathrm{~s}, 63^{\circ} \mathrm{C}$ for $20^{\circ} \mathrm{C}$, and $72^{\circ} \mathrm{C}$ for $30 \mathrm{~s}$, with a final step at $72^{\circ} \mathrm{C}$ for $10 \mathrm{~min}$ (Antikainen et al., 2009). Furthermore, a multiplex 15-plex PCR was performed to identify VF associated with UPEC; 15 pairs of primers were used to detect $15 \mathrm{VF}$ genes [papAH, PapG (allele I), PapG (allele II), PapG (allele III), fimH, afa/ draBC, sfa/focDE, hlyA, cnf1, iutA, fyuA, kpsMII, traT, ibeA, malX, PAI] as described (Moreno et al., 2005). To facilitate interpretation of the gel images, the primers were separated into 2 pools. Pool A was composed of 8 pairs of primers (Allele III f, Allele III r, Afa f, Afa r, sfa 1, sfa 2, hlyA f, hlyA r, cnf1, cnf2, FyuA f, FyuA r, TraT f, TraT r, Ibe10 f, and Ibe10 r), and Pool B was composed of 7 pairs of primers (PapA f, PapA r, Allele I f, Allele I r, Allele II f, Allele II r, FimH f, FimH r, AerJ f, AerJ r, kps II f, kps II r, RPAi f, and RPAi r). The PCR of pools A and B were conducted separately; the thermal cycling protocol was $95^{\circ} \mathrm{C}$ for $12 \mathrm{~min}$, followed by 25 cycles at $94^{\circ} \mathrm{C}$ for $30 \mathrm{~s}, 63^{\circ} \mathrm{C}$ for $30 \mathrm{~s}$, and $68^{\circ} \mathrm{C}$ for $3 \mathrm{~min}$, followed by a final extension at $72^{\circ} \mathrm{C}$ for $10 \mathrm{~min}$ (Moreno et al., 2005).

\section{DNA Gyrase Amplification, Sequencing, and Phylogenetic Analysis}

For this molecular analysis, only genetically distinct bacteria (based on RAPD-PCR gels) presenting at least one of the following combinations of $\mathrm{VF}$ genes were used: fimH and hlyA, fimH and ibeA, fimH and kpsMII, fimH and $c d t$, and fimH and astA. Chromosomal DNA was extracted with InstaGene Matrix (Bio-Rad Laboratories) and amplified by PCR in a 2720 Thermal Cycler (Applied Biosystems). The PCR conditions and primers used were as described previously (Fukushima et al., 2002). The purified PCR products were sequenced using DNA Illumina Paired-End sequencing at the Cornell University Life Sciences Core Laboratory Center (CLC, Ithaca, NY). The PCR fragments were determined by using the sequencing primers UP1S (5'-GAAGTCATCATGACCGTTCTGCA-3') and UP2Sr (5'-AGCAGGGTACGGATGTGCGAGCC-3') (Fukushima et al., 2002). For phylogenetic analysis, DNA gyrase $($ gyr B $)$ sequences from 41 intrauterine $E$. coli (IUEC) genetically distinct bacteria were aligned with 1 Escherichia fergusonii strain, 5 Salmonella enteric ssp. enteric strains, 1 Shigella flexneri, 1 Shigella dysenteriae, 2 Shiguella boydii, and 33 E. coli strains.
The gyrB sequences were aligned using the Clustal-W algorithm using the software Geneious 4.8.4 (Biomatters Ltd., Auckland, New Zealand) and adjusting for 1,050 bases. All bacterial strains used in this phylogenetic analysis, with the exception of the IUEC, have had their whole chromosomal genomes sequenced and were publically available at the National Center for Biotechnology Information (NCBI) website (http://www.ncbi. nlm.nih.gov/) at the time the analysis was performed. Detailed information regarding E. coli pathotype and strain identification is included in the phylogenetic tree. Evolutionary relationships were inferred by using the neighbor-joining method, and the evolutionary distances were computed by using the Tamura-Nei method (Tamura and Nei, 1993). Bootstrap values were calculated from 1,000 replicate analyses.

\section{Statistical Analysis}

Hierarchical cluster analysis was performed using the RAPD-PCR agarose gel pattern data from one randomly selected $E$. coli isolate per culture-positive cow. Hierarchical cluster analysis was done using the Ward method of the statistical software JMP (SAS Institute Inc., Cary, NC). For subsequent analysis the research unit was the cow. To facilitate data analysis and interpretation, a new 3-level variable was created for each of the 32 investigated VF. Cows were classified as 0 when they were culture-negative, 1 when the cow was culture-positive but the VF was absent in all E. coli isolates, and 2 when the culture was positive and the VF factor was present in at least one $E$. coli isolate.

Partition analysis was performed in JMP and for this analysis the dependent variable in the model was the dichotomous variable metritis (0 or 1$)$ and the independent variables were birth (singleton or twin), calving (assisted or unassisted), and parity group (1 to 3). Several logistical regression models were performed in Stata (StataCorp LP, College Station, TX) to assess the effect of the VF on the probability of metritis and endometritis. The dependent variables in these models were metritis (yes or no) and endometritis (yes or no), and the independent variables were the identified VF, parity group (1 to 3 ), and farm. Adjusted probabilities and respective $95 \%$ confidence intervals were estimated from the logistic regression equations by using the predicted probability option in Stata. A similar logistic regression model was used to analyze the effect of BCS as defined by Edmonson et al. (1989), status of the newborn calf (twin, stillborn, male alive, female alive), retained placenta ( 0 or 1 ), and calving ease (assisted and unassisted) on the odds of intrauterine E. coli contamination. Two-way interactions between the independent variable of interest VF and all the 
other independent variables were tested; however, no significant interactions were encountered. A stratified analysis was performed to assess the interaction of the VF ast $A, c d t, k p s M I I, i b e A$, and hlyA with fim $H$ on the probability of metritis using the categorical modeling option of JMP.

To assess the effect of VF on calving to conception interval, a Cox proportional hazards model was fitted to the data using the proportional hazard regression procedure in SAS (SAS Institute Inc., Cary, NC). For analysis of reproduction, cows were right-censored if not diagnosed as being pregnant before culling, death, or at the end of the data collection period. Variables included in the model were parity number and the VF cdt, astA, hlyA, ibeA, kpsMII, and fimH.

The variables $V F$ had 3 levels ( 0 when they were culture-negative, 1 when the cow was culture-positive but the VF was absent in all E. coli isolates, and 2 when the culture was positive and the VF factor was present in at least one E. coli isolate), and 3 regression models were used to evaluate the effect of the VF on the odds of metritis and endometritis as well as the hazard of pregnancy. In all regression models the reference level used was level $=0$, which represents the culture negative cows. This level was chosen to be the reference because a consistent increasing biological gradient was observed between the comparisons of level 1 versus 0 and level 2 versus 0 . Additionally, adjusted confidence intervals for the incidence of metritis and endometritis, as well as for the hazard of pregnancy, were calculated using the regression parameters for each of the 3 levels of the significant VF. Therefore, statistical comparison between all levels of the VF variables is made possible by comparing their respective parameter estimates and confidence intervals.

\section{RESULTS}

\section{Bacterial Isolation, Characterization, and RAPD-PCR Cluster Analysis}

A total of 374 cows were enrolled in the study, of which $33.4 \%$ were positive for $E$. coli culture. Five $E$. coli were isolated from each of the 125 positive cows, totaling an initial pool of isolates of 625 . All isolates (n $=625$ ) were confirmed to be $E$. coli by cultural methods using chromogenic agar (CHROMagar). However, 14 isolates were negative for rDNA amplification and were excluded from the study. DNA fingerprinting using RAPD-PCR was performed for all remaining 611 isolates and revealed that, on average, 2.8 genetically distinct $E$. coli isolates were found per contaminated cow. A cluster analysis was performed using the DNA fingerprinting information from the RAPD-PCR aga- rose gels. The analysis was performed with the number of clusters set to 8 . The RAPD-PCR banding pattern appeared to have an association with the pathogenicity of the E. coli isolates, because significant variation existed in the probability of metritis among the 8 different clusters; metritis incidence varied from $67 \%$ in cluster 3 $(\mathrm{n}=3)$ to $18 \%$ in cluster $5(\mathrm{n}=11$; Figure 1$)$. Bacterial isolates did not significantly cluster farm of origin (data not shown). The RAPD-PCR agarose gel images were evaluated, and only genetically distinct isolates within each cow were selected for further PCR work.

\section{Analysis of Risk Factors for Intrauterine E. coli Contamination}

For this analysis, a logistic regression model was fitted to the data to analyze the effect of BCS and the status of the calf at birth on the odds of intrauterine $E$. coli contamination. Cows that had twin parturitions were 4.4 times more likely to have intrauterine $E$. coli contamination compared with cows that gave birth to live female calves $(P<0.01$; Table 1$)$. Stillborn parturitions and parturitions to live male calves also increased the odds of intrauterine contamination by E. coli (3.7-fold and 1.6-fold, respectively) compared with parturition to live female calves $(P<0.01$; Table 1$)$. Furthermore, low BCS at uterine swab sampling was associated with increased odds of intrauterine contamination by $E$. coli. The odds of intrauterine E. coli contamination were 2.8 and 2.3 times higher for cows with $\mathrm{BCS}=3$ and BCS $<3$ compared with cows with BCS $>3(P<0.01)$. The variable calving (assisted vs. unassisted) was not significant and was dropped from the model. The variable retained placenta was significant; cows with retained placenta were 5 times more likely to have intrauterine E. coli contamination than nonretained placenta cows.

\section{Association of E. coli VF with the Risk of Postpartum Metritis}

Postpartum metritis was diagnosed in $19.5 \%$ of all cows enrolled in the study $(\mathrm{n}=374)$. For this analysis, the research unit was the cow, classified into 1 of 3 categories: 0 when they were culture-negative, 1 when the cow was culture-positive but the VF was absent in all $E$. coli isolates, and 2 when the culture was positive and the VF was present in at least one $E$. coli isolate. Of the $32 \mathrm{VF}$ evaluated, only 11 were identified in the studied collection of E. coli: cdt, astA, hlyA, fyfA, traT, Pap $G$ alleles 1 and 2, ibeA, papAH, kpsMII, and fimH. Of the identified VF, only 6 were significantly associated with metritis: fimH, astA, cdt, kpsMII, ibeA, and hlyA (Table 2). 
Cluster 1:

$48 \%$ metritis $(n=25)$

Cluster 6:

$31 \%$ metritis $(n=16)$

Cluster 4:

$22 \%$ metritis $(n=23)$

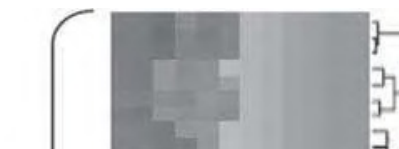

$23 \%$ metritis $(n=18)$

$38 \%$ metritis $(n=13)$

Cluster 7:

$55 \%$ metritis $(\mathrm{n}=11)$

Cluster 5:

$18 \%$ metritis $(\mathrm{n}=11)$

Cluster 3:

$67 \%$ metritis $(n=3)$

Figure 1. Randomly amplified polymorphic DNA (RAPD)-PCR dendrogram. One Escherichia coli isolate per cow was randomly selected for use in this analysis; 120 bacterial isolates from 120 different cows were used. Cluster numbers and respective metritis incidences are also presented.

A partition analysis was performed to estimate the ranking of all VF and risk factors analyzed, and fimH was found to be the strongest predictor of metritis (Figure 2). This can be explained by the high prevalence of the fimH gene in IUEC $(87 \%, \mathrm{n}=125)$ and because of the higher probability of metritis in the presence of fim $H$ genes: cows with at least $1 \mathrm{fimH}$-carrying IUEC isolate had 6.0-fold greater odds of metritis compared 
Table 1. Logistic regression analysis assessing the effect of several risk factors on the odds of intrauterine contamination by Escherichia coli

\begin{tabular}{lcccc}
\hline & \multicolumn{5}{c}{$\begin{array}{c}\text { E. coli } \\
\text { contamination } \\
\text { adjusted } \% \\
(95 \% \text { CI })\end{array}$} & $\begin{array}{c}\text { Adjusted } \\
\text { odds ratio }\end{array}$ & $P$-value \\
Risk factor & $\mathrm{n}$ & $60(35-80)$ & 4.4 & $<0.01$ \\
\hline Twin & 18 & $55(32-77)$ & 3.7 & \\
Stillborn & 18 & $35(27-32)$ & 1.6 & \\
Male alive & 138 & $25(19-31)$ & Ref. & \\
Female alive & 200 & $36(26-47)$ & 2.3 & $<0.01$ \\
BCS $<3$ & 142 & $42(32-52)$ & 2.8 & \\
BCS $=3$ & 98 & $20(13-29)$ & Ref. & \\
BCS $>3$ & 134 & $29(24-34)$ & Ref & $<0.01$ \\
Nonretained placenta & 339 & $65(44-81)$ & 4.7 & \\
Retained placenta & 35 & &
\end{tabular}

${ }^{1}$ Referent category.

with culture-negative cows. Moreover, cows that were positively cultured for IUEC carrying the $c d t$, ast A, or hlyA gene were $6.7,12$, and 10.5 times more likely, respectively, to have metritis than cows that did not present uterine contamination (Table 2).

To further understand the interaction of astA, cdt, kpsMII, ibeA, and hlyA with fimH on the probability of metritis, a stratified descriptive analysis was performed (Figure 3). When the VF fimH was absent, the incidence of metritis was low $(<24 \%)$, even in the presence of other VF. However, the incidence of metritis for cows contaminated with a fimH-carrying IUEC was equally high in the absence of all other VF. Additionally, a synergetic relationship was observed between fim $H$ and ast $A, c d t$, kpsMII, ibe A, or hlyA, because the incidence of metritis was highest $(>52 \%)$ when fimH occurred concurrently with 1 of those 5 VF (Figure 3).

\section{Association of E. coli VF with the Risk of Clinical Endometritis}

For this analysis only a subset of the cows were used $(\mathrm{n}=117)$ and the observed overall prevalence of en-

Table 2. Association of known Escherichia coli virulence factors with the risk of postpartum metritis. The confounding variables farm and parity number were added to all models

\begin{tabular}{|c|c|c|c|c|}
\hline Virulence factor & $\mathrm{n}$ & $\begin{array}{c}\text { Metritis } \\
\text { adjusted } \\
\text { incidence } \\
(95 \% \mathrm{CI})\end{array}$ & $\begin{array}{l}\text { Adjusted } \\
\text { odds ratio }\end{array}$ & $P$-value \\
\hline Culture negative & 249 & $11(8-15)$ & Ref. $^{1}$ & \multirow[t]{3}{*}{$<0.001$} \\
\hline fimH absent & 16 & $22(18-27)$ & 2.6 & \\
\hline fimH present & 109 & $39(30-49)$ & 4.6 & \\
\hline Culture negative & 249 & $11(8-15)$ & Ref. & \multirow[t]{3}{*}{$<0.001$} \\
\hline ast $A$ absent & 102 & $28(24-38)$ & 3.7 & \\
\hline ast $A$ present & 23 & $63(46-74)$ & 10.2 & \\
\hline Culture negative & 249 & $11(8-15)$ & Ref. & \multirow[t]{3}{*}{$<0.001$} \\
\hline$i b e A$ absent & 101 & $29(24-36)$ & 4.1 & \\
\hline$i b e A$ present & 24 & $57(40-72)$ & 8.4 & \\
\hline Culture negative & 249 & $11(8-15)$ & Ref. & \multirow[t]{3}{*}{$<0.001$} \\
\hline$c d t$ absent & 93 & $28(22-35)$ & 4.0 & \\
\hline$c d t$ present & 32 & $46(39-67)$ & 6.7 & \\
\hline Culture negative & 249 & $11(8-15)$ & Ref. & \multirow[t]{3}{*}{$<0.001$} \\
\hline hly $A$ absent & 109 & $31(25-39)$ & 4.7 & \\
\hline hly $A$ present & 16 & $63(47-76)$ & 9.7 & \\
\hline Culture negative & 249 & $11(8-15)$ & Ref. & \multirow[t]{3}{*}{$<0.001$} \\
\hline kpsMII absent & 109 & $31(24-38)$ & 4.7 & \\
\hline kpsMII present & 16 & $61(45-74)$ & 9.4 & \\
\hline
\end{tabular}

${ }^{1}$ Referent category. 


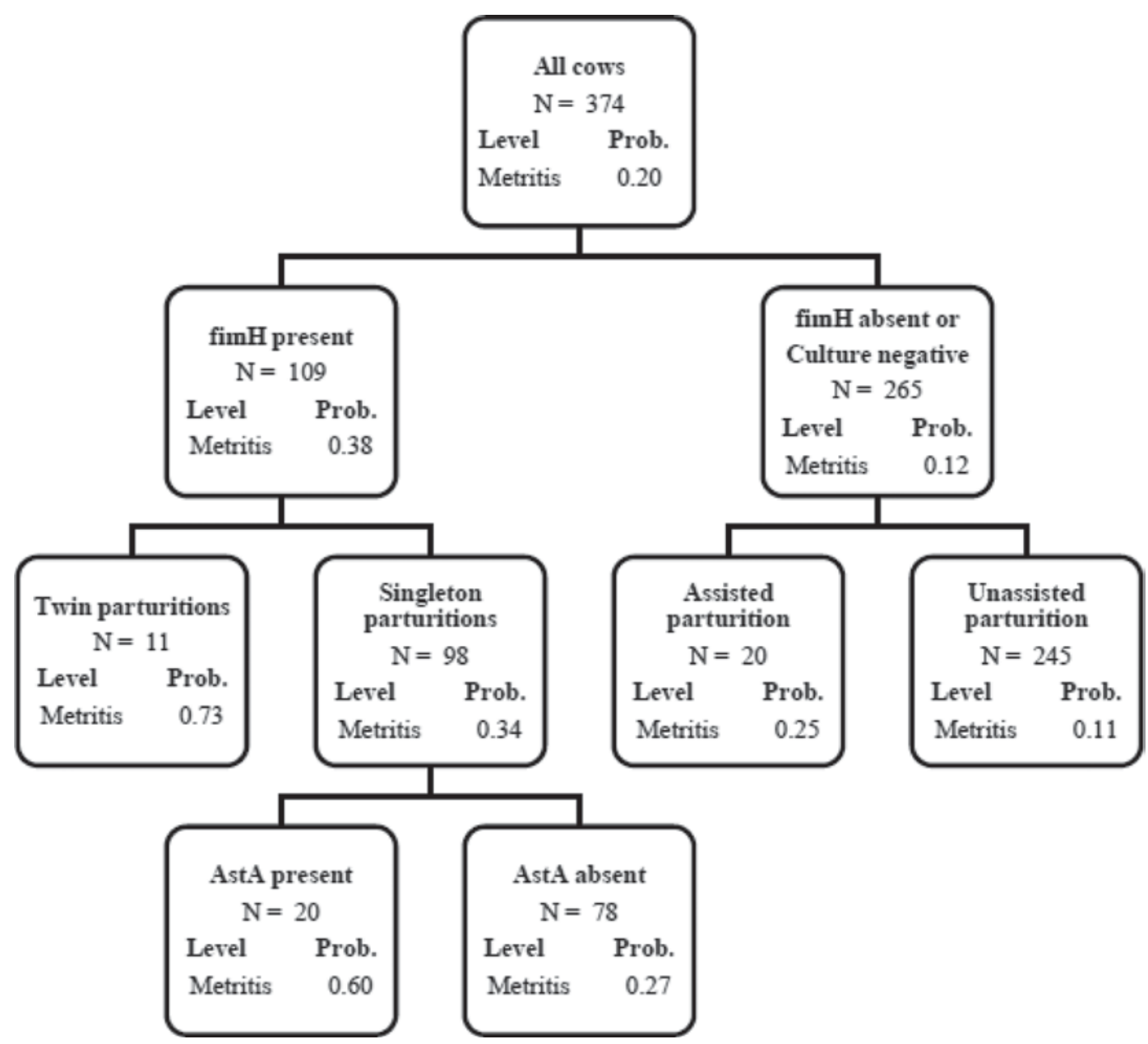

Figure 2. Partition analysis tree illustrating the hierarchical order of the risk factors for metritis. The dependent variable in the model was the dichotomous variable metritis, and the independent variables were birth (singleton or twin), calving (assisted or unassisted), and parity (primiparous or multiparous). Additionally, the phylogenetic groupings (A, B1, B2, D) of all tested virulence factors were offered to the model as independent variables. The tree indicates that the virulence factor fim $H$ was the most important predictor of metritis, and the variables birth, calving, and the virulence factor ast $A$ were the second, third, and fourth most relevant predictors, respectively.

dometritis was $47 \%$. Further analysis was performed on fimH, ast A, cdt, kpsMII, ibeA, and hlyA to evaluate their association with the incidence of clinical endometritis. Cows positive for IUEC carrying fimH, cdt, and ast $A$ genes had, respectively, 2.6, 3.7, and 4.6 times increased odds of having clinical endometritis compared with cows that did not present uterine contamination (Table 3).

\section{Association of E. coli VF with Reproductive Parameters}

Culture-positive cows with a fimH-carrying IUEC were at a 1.8 times increased hazard of being detected pregnant compared with cows with no uterine contamination (Table 4). Cows with uterine contamination by an IUEC carrying the $c d t$ or $k p s M I I$ gene were 2.3 and
9.2 times, respectively, less likely to become pregnant than cows that did not have uterine contamination (Table 4).

\section{DNA Gyrase Phylogenetic Analysis}

For this analysis, the DNA gyrase sequences from 41 IUEC genetically distinct bacteria were aligned with 1 Escherichia fergusonii strain, 5 Salmonella enteric ssp. enteric strains, 1 Shigella flexneri, 1 Shigella dysenteriae, 2 Shigella boydii, and 33 E. coli strains. Figure 4 shows the phylogenetic tree for these species based on the gyrB gene sequence. As expected, all Salmonella were grouped into the same clade, apart from the large clade of the E. coli. All E. coli form the O157:H7 serogroup grouped together and their $\operatorname{gyr} B$ sequences were close to identical; 4 IUEC also grouped with the O157:H7 


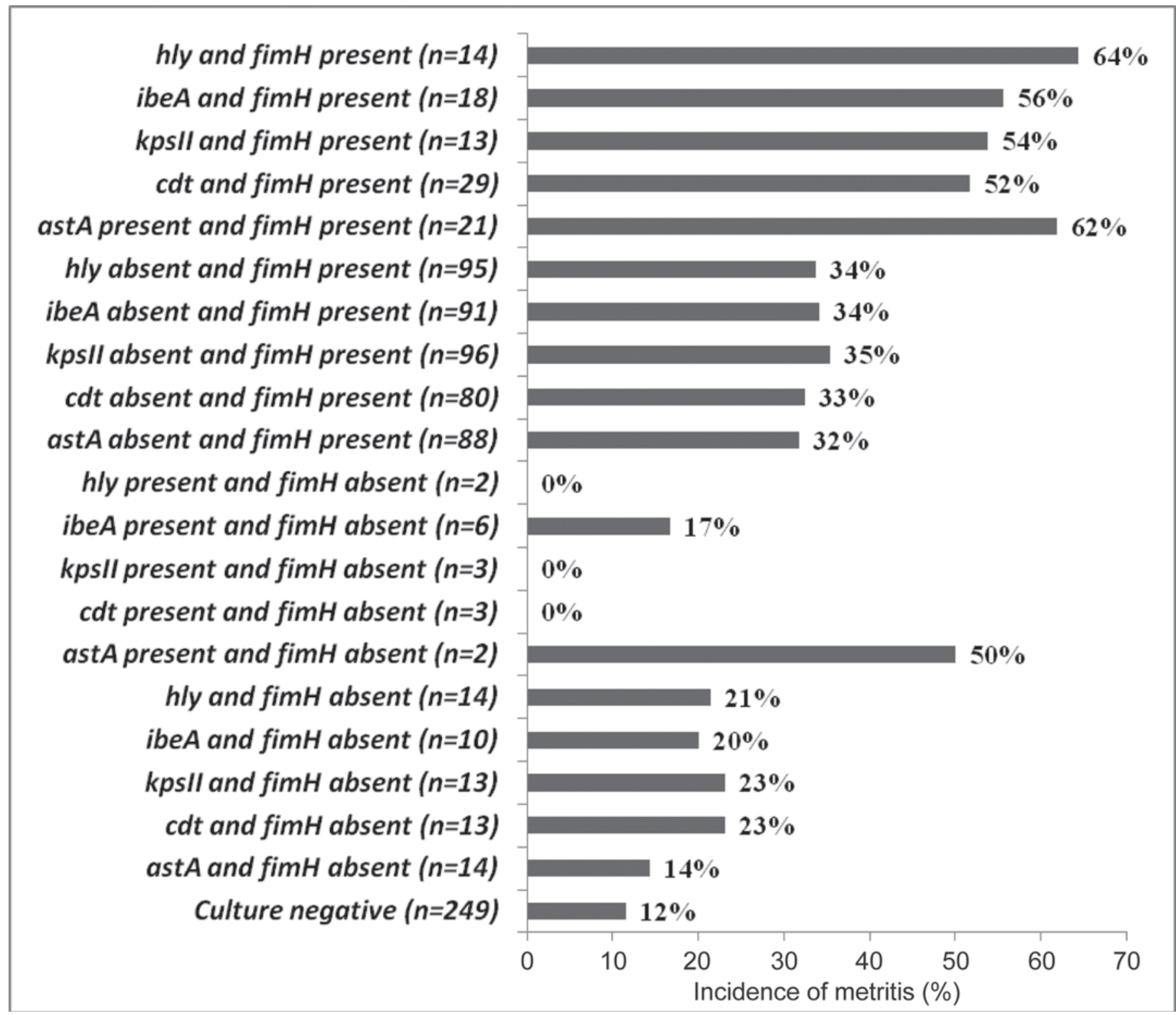

Figure 3. Graphical illustration of the interaction of virulence factors ast $A, c d t, k p s M I I$, ibeA, and $h l y A$ with fim $H$ on the incidence of metritis. Only culture-positive cows were used for this analysis.

E. coli. All reference extraintestinal pathogenic E. coli (ExPEC) grouped in the same clade, and 9 IUEC were grouped with the ExPEC. The majority of the IUEC (n $=23$ ) were grouped in an almost unique clade adjacent to the ExPEC.

\section{DISCUSSION}

The present study demonstrated that IUEC was associated with puerperal uterine infection. Dairy cows infected with IUEC in the early postpartum period (3-7 d postpartum) were at higher risk of developing puerperal metritis and clinical endometritis at around $10 \mathrm{~d}$ and $35 \mathrm{~d}$ postpartum, respectively. Because of IUEC uterine infection, the reproductive performance of the affected cows was severely decreased.

Several cow-related factors are known to increase the risk of metritis by facilitating access of environmental bacteria into the uterine lumen (e.g., assisted parturition and retained placenta; Benzaquen et al., 2007) or by allowing excessive growth of bacteria within the uterine lumen (e.g., twin parturition and suppressed immune system; Sheldon et al., 2006). In the present study, we observed that the status of the newborn calf (twin, still- 
Table 3. Association of known Escherichia coli virulence factors with the risk of postpartum endometritis. The confounding variables farm and parity number were added to all models

\begin{tabular}{|c|c|c|c|c|}
\hline Virulence factor & $\mathrm{n}$ & $\begin{array}{c}\text { Endometritis } \\
\text { adjusted } \\
\%(95 \% \text { CI })\end{array}$ & $\begin{array}{c}\text { Adjusted } \\
\text { odds ratio }\end{array}$ & $P$-value \\
\hline Culture negative & 80 & $40(31-52)$ & Ref. ${ }^{1}$ & \multirow[t]{3}{*}{0.04} \\
\hline fim $H$ absent & 6 & $51(41-62)$ & 0.71 & \\
\hline fim $H$ present & 31 & $62(44-78)$ & 2.6 & \\
\hline Culture negative & 80 & $40(31-52)$ & Ref. & \multirow[t]{3}{*}{0.05} \\
\hline ast $A$ absent & 24 & $56(43-68)$ & 1.3 & \\
\hline ast $A$ present & 13 & $70(47-86)$ & 4.6 & \\
\hline Culture negative & 80 & $40(31-52)$ & Ref. & \multirow[t]{3}{*}{0.06} \\
\hline$i b e A$ absent & 28 & $57(43-70)$ & 1.6 & \\
\hline$i b e A$ present & 9 & $72(46-88)$ & 4.8 & \\
\hline Culture negative & 80 & $40(31-52)$ & Ref. & \multirow[t]{3}{*}{0.05} \\
\hline$c d t$ absent & 26 & $55(42-68)$ & 1.6 & \\
\hline$c d t$ present & 11 & $69(44-86)$ & 3.7 & \\
\hline Culture negative & 80 & $40(31-52)$ & Ref. & \multirow[t]{3}{*}{0.06} \\
\hline hly $A$ absent & 32 & $58(43-71)$ & 1.8 & \\
\hline hlyA present & 5 & $73(44-90)$ & 5.5 & \\
\hline Culture negative & 80 & $40(31-52)$ & Ref. & \multirow[t]{3}{*}{0.07} \\
\hline kpsMII absent & 30 & $56(42-70)$ & 1.8 & \\
\hline kpsMII present & 7 & $71(43-88)$ & 3.9 & \\
\hline
\end{tabular}

${ }^{1}$ Referent category.

born, and sex) significantly affected the probability of intrauterine E. coli contamination. For example, cows that had twin parturitions were 4.4 times more likely to be infected with an IUEC. Previous epidemiological research from our group has demonstrated that twin- ing, calf weight, and stillborn parturition significantly decreased reproductive efficiency and cow survivability (Bicalho et al., 2007a,b; Linden et al., 2009). The present study adds important new insight into how calvingrelated factors are associated with poor reproductive

Table 4. Cox proportional hazards analysis evaluating the effect of the 6 significant virulence factor genes (fimH, ast A, cdt, kpsMII, ibeA, and hlyA) on the calving-to-conception interval

\begin{tabular}{|c|c|c|c|c|c|}
\hline Variable & $\mathrm{n}$ & $\begin{array}{c}\text { Hazard } \\
\text { ratio }\end{array}$ & & $\begin{array}{c}95 \% \\
\text { CI }\end{array}$ & $P$-value \\
\hline $\begin{array}{l}\text { Culture negative } \\
c d t \text { absent } \\
c d t \text { present }\end{array}$ & $\begin{array}{r}249 \\
93 \\
32\end{array}$ & $\begin{array}{l}1.6 \\
2.3\end{array}$ & Ref. ${ }^{1}$ & $\begin{array}{l}1.1-2.4 \\
1.1-4.7\end{array}$ & 0.007 \\
\hline $\begin{array}{l}\text { Culture negative } \\
\text { ast } A \text { absent } \\
\text { ast } A \text { present }\end{array}$ & $\begin{array}{r}249 \\
102 \\
23\end{array}$ & $\begin{array}{l}1.7 \\
2.2\end{array}$ & Ref. & $\begin{array}{l}1.1-2.5 \\
1.0-5.0\end{array}$ & 0.007 \\
\hline $\begin{array}{l}\text { Culture negative } \\
\text { hlyA absent } \\
\text { hlyA present }\end{array}$ & $\begin{array}{r}249 \\
109 \\
16\end{array}$ & $\begin{array}{l}1.7 \\
3.1\end{array}$ & Ref. & $\begin{array}{l}1.1-2.4 \\
1.0-9.8\end{array}$ & 0.006 \\
\hline $\begin{array}{l}\text { Culture negative } \\
\text { ibeA absent } \\
\text { ibeA present }\end{array}$ & $\begin{array}{r}249 \\
101 \\
24\end{array}$ & $\begin{array}{l}1.9 \\
1.4\end{array}$ & Ref. & $\begin{array}{l}1.3-2.8 \\
0.7-2.7\end{array}$ & 0.007 \\
\hline $\begin{array}{l}\text { Culture negative } \\
\text { kpsMII absent } \\
\text { kpsMII present }\end{array}$ & $\begin{array}{r}249 \\
109 \\
16\end{array}$ & $\begin{array}{l}1.6 \\
9.2\end{array}$ & Ref. & $\begin{array}{l}1.1-2.3 \\
1.3-66\end{array}$ & $<0.001$ \\
\hline $\begin{array}{l}\text { Culture negative } \\
\text { fimH absent } \\
\text { fimH present }\end{array}$ & $\begin{array}{r}249 \\
16 \\
109\end{array}$ & $\begin{array}{l}1.7 \\
1.8\end{array}$ & Ref. & $\begin{array}{l}0.7-3.9 \\
1.2-2.6\end{array}$ & 0.006 \\
\hline
\end{tabular}

${ }^{1}$ Referent category. 


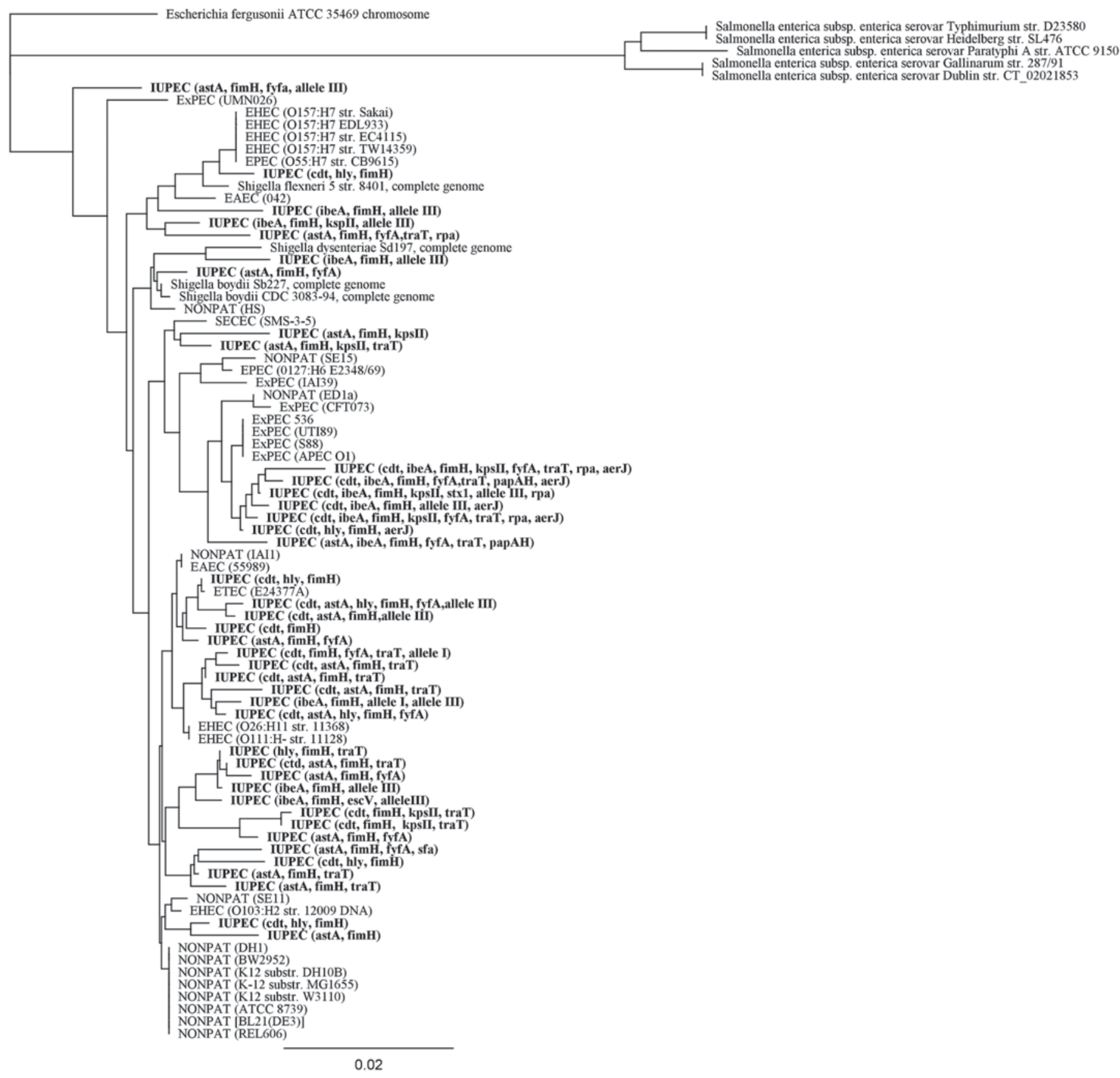

Figure 4. Phylogenetic tree based on DNA gyrase B $(\operatorname{gyr} B)$ sequences illustrating the evolutionary distance between 41 genetically distinct strains of intrauterine Escherichia coli (IUEC), 33 strains of E. coli with known pathotypes, 4 Shigella, and 5 strains of Salmonella. ExPEC = extraintestinal E. coli; EAEC = enteroaggregative E. coli; IUPEC = intrauterine pathogenic E. coli; EHEC = enterohemorrhagic E. coli; NONPAT $=$ nonpathogenic E. coli $\mathrm{SECEC}=E$. coli strain SMS-3-5; EPEC = enteropathogenic E. coli; ETEC = enterotoxigenic $E$. coli.

efficiency, by associating newborn calf status with probability of IUEC infection.

The significance of $E$. coli in the pathogenesis of puerperal uterine infection is poorly understood and few studies have attempted to elucidate the etiological role of $E$. coli in this process. Recently, a collection of cattle uterine $E$. coli strains was screened for the presence of
$15 \mathrm{VF}$ genes and none was found to be associated with uterine disease (Silva et al., 2009). The authors concluded that uterine $E$. coli were merely opportunistic environmental bacteria, because none of the evaluated VF appeared to affect the probability of uterine infection. In contrast, the present study evaluated $32 \mathrm{VF}$, of which 6 were significantly associated with the inci- 
dence of metritis and endometritis. The identification of the VF genes fimH, astA, cdt, kpsMII, ibeA, and hlyA increased the risk of uterine disease and reproductive failure, and these significant VF were not evaluated by Silva et al. (2009). Sheldon et al. (2010) evaluated the effect of $17 \mathrm{VF}$ genes and none were found to be associated with uterine disease. Of the 6 significant VF (fimH, astA, cdt, kpsMII, ibeA, and hlyA) found in the present study, ibe $A$ was the only VF that was also assessed by Sheldon et al. (2010) and none of the isolates in that study were $i b e A$-positive. However, in the present study, 374 cows and 625 bacterial isolates were analyzed, which is a much larger sample size compared with the previous studies (Silva et al., 2009; Sheldon et al., 2010).

In humans, E. coli is a major cause of urinary tract infection (UTI), estimated to be the primary cause of $>80 \%$ of community-acquired UTI (Struelens et al., 2004). Human UTI is likely to be initiated by contamination of the urethra and bladder by fecal E. coli, including uropathogenic E. coli (UPEC) and commensal flora. Hours after contamination, the bladder environment will quickly select for bacteria expressing type 1 fimbriae, and tissue colonization and damage follow (Kaper et al., 2004). In the present study, fimH was highly prevalent in E. coli-infected cows and was an important predictor of metritis and endometritis. Type 1 pili containing the fimH adhesin are present in virtually all UPEC, and fimH immunization in a murine model prevented in vivo colonization of the bladder mucosa by $99 \%$ (Langermann et al., 1997). In bovine metritis, recent research indicates that the infected uterus is predominated by E. coli in the first week postpartum, which alters the intrauterine environment to support future infection by other opportunistic anaerobic bacteria starting in the second week postpartum (Dohmen et al., 2000; Zerbe et al., 2001). Sheldon et al. (2010) concluded that endometrial pathogenic E. coli were expressing the fim $\mathrm{H}$ gene, because mannose treatment of $E$. coli isolates decreased their ability to adhere to endometrial cells.

The group of ExPEC is diverse and includes several pathotypes, including UPEC, neonatal meningitis $E$. coli, avian pathogenic E. coli (APEC), necrotoxigenic E. coli, and the newly described IUEC. All ExPEC will encounter similar challenges during the process of establishing extraintestinal infections, and consequently, they are likely to share similar VF genes (Johnson et al., 2008). The IUEC bacteria described in this study presented numerous relationships with human ExPEC and APEC. The VF gene ibeA (invasion of brain endothelium) plays an important role in neonatal gramnegative meningitis in humans, which is mainly caused by vertical transmission of $E$. coli (Huang et al., 2001).
It has been suggested that $i b e A$ contributes to the invasiveness of $E$. coli into brain microvascular endothelial cells via a ligand-receptor interaction (Huang et al., 2001). It is possible that such a ligand-receptor interaction can be facilitated by ibe $A$ expression in mammalian tissues other than brain microvascular endothelial cells, such as the endometrium. It has also been reported that ibeA plays an important role in the pathogenesis of extraintestinal infection of birds by APEC. Germon et al. (2005) demonstrated that the virulence of an $i b e A$ free mutant APEC was reduced compared with APEC expressing $i b e A$. The prevalence of $i b e A$ in the present study was $19.2 \%$, which is not very different from the prevalence found in APEC (26\%; Germon et al., 2005), neonatal meningitis E. coli $(33-40 \%)$, and vaginal isolates (32\%; Johnson et al., 2001; Obata-Yasuoka et al., 2002).

The VF gene kpsMII encodes the capsule proteins K1 or K5 and has been associated with cellulitis in chickens (de Brito et al., 2003) and UTI in women (Moreno et al., 2005, 2009). In the present study, kpsMII was found in $12.8 \%$ of the E. coli-positive cows, which is comparable to the $16 \%$ prevalence found in chickens with cellulitis (de Brito et al., 2003) and the $21 \%$ found in UTI in humans (Johnson et al., 2002). Another significant VF gene encountered in the present study encodes hly, which is a heat-labile extracellular protein synthesized by a large proportion of ExPEC isolates (Smith et al., 2008). The hly toxin is responsible for poring the membrane and lysing several different mammalian cells (Lally et al., 1999). A total of $12.8 \%$ of the E. coli-positive cows in this study were carriers of bacteria with the hlyA gene.

The partition analysis performed in this study revealed the $\mathrm{VF}$ gene ast $A$ to be an important predictor of metritis, second only to fimH. A total of $18.5 \%$ of the E. coli-positive cows were infected with an astApositive IUEC. Cows with an astA-carrying IUEC were 12 times more likely to develop postpartum metritis and 4.6 times more likely to develop endometritis compared with $E$. coli-negative cows. The ast $A$ gene is an important characteristic of enteroaggregative $E$. coli (EAEC), which are a common cause of diarrhea in humans, especially childhood diarrhea in the developing world; however, more recently, EAEC have also been associated with foodborne infections in industrialized countries (Nataro et al., 1998). The astA gene encodes a 38-AA protein named enteroaggregative $E$. coli heatstable enterotoxin 1 (EAST1; Veilleux and Dubreuil, 2006). The specific role of EAST1 in the pathogenesis of EAEC-related diarrhea is not completely understood (Veilleux and Dubreuil, 2006). Although, astA is typically detected in EAEC, it has also been detected in other $E$. coli pathotypes such as enterohemorrhagic $E$. 
coli, enteropathogenic E. coli, and enterotoxigenic $E$. coli as well as other bacterial species such as Salmonella (Paiva de Sousa and Dubreuil, 2001). Abe et al. (2008) demonstrated that UPEC can carry VF genes from diarrheagenic E. coli, especially $\mathrm{VF}$ associated with EAEC. It is unknown whether ExPEC strains have acquired EAEC genes or whether some EAEC are involved in extraintestinal infections such as UTI (Abe et al., 2008). Nevertheless, our study suggests that EAST1 may play an important role in the pathogenesis of postpartum uterine infections.

A multiplex PCR for the detection of genes encoding cytolethal distending toxin (CDT) and capable of detecting CDT-I, CDT-II, CDT-III, and CDT-IV was also performed. A total of $25.6 \%$ of the E. coli-positive cows were $c d t$-positive, and when the $c d t$ gene was detected in conjunction with the fim $H$ gene, $52 \%$ of the cows developed postpartum metritis compared with only $12 \%$ for the culture-negative cows. Cytolethal distending toxins comprise a unique family of toxins that cause characteristic enlargement of specific mammalian cells followed by death of those cells (De Rycke and Oswald, 2001). Although $c d t$ was initially identified in an enteropathogenic $E$. coli, it is now known that $c d t$ is widely present in other $E$. coli pathotypes, mainly in ExPEC (Toth et al., 2003).

Finally, the DNA gyrase sequences from 41 genetically distinct IUEC were aligned with a variety of enteric bacteria, including $33 \mathrm{E}$. coli reference strains. The phylogenetic tree (Figure 4) revealed that the IUEC fall into 2 major clades: a clade of all ExPEC and a clade mainly composed of IUEC that is adjacent to the ExPEC clade. It has been suggested that use of the gyrB gene for phylogenetic analysis of closely related bacterial strains is preferred over the use of the $16 \mathrm{~S}$ rRNA region because the $\operatorname{gyr} B$ gene evolves at a higher rate, providing more heterogeneity for the analysis (Fukushima et al., 2002). Those authors suggested that the $\operatorname{gyr} B$ region could have high reliability for the identification of pathogenic bacteria.

It is important to acknowledge that, in the present study, isolation of bacterial species other than E. coli was not attempted, and it is possible that uterine disorders are caused by a mixed infection and that further research is needed to complete our knowledge regarding the etiology of metritis and the role played by E. coli. Nevertheless, the identification of E. coli VF genes associated with metritis and endometritis is important evidence toward considering $E$. coli as a primary uterine infection pathogen. Additionally, our study was a large population-based study with several hundred cows enrolled, and several logistic regression models were used to analyze 11 different VF genes, which could have favored the occurrence of a type 1 statistical error.

\section{CONCLUSIONS}

In summary, IUEC were associated with postpartum metritis and endometritis. Additionally, several similarities between ExPEC and IUEC were identified, such as the presence of the VF genes fimH, ast A, cdt, kpsMII, $i b e A$, and $h l y A$ and their association with metritis and endometritis. An important EAEC virulence factor (astA) was also prevalent among the IUEC isolates and when present it increased the chances of metritis significantly. Therefore, 6 important VF genes were associated with metritis and endometritis: fim $H$, ast $A$, cdt, kpsMII, ibeA, and hlyA. The fimH gene was the most prevalent $\mathrm{VF}$ and the most significant. The VF $a s t A, c d t$, kpsMII, ibeA, and hlyA were highly pathogenic when detected in conjunction with fim $H$.

\section{REFERENCES}

Abe, C. M., F. A. Salvador, I. N. Falsetti, M. A. Vieira, J. Blanco, J. E. Blanco, M. Blanco, A. M. Machado, W. P. Elias, R. T. Hernandes, and T. A. Gomes. 2008. Uropathogenic Escherichia coli (UPEC) strains may carry virulence properties of diarrhoeagenic E. coli. FEMS Immunol. Med. Microbiol. 52:397-406.

Antikainen, J., E. Tarkka, K. Haukka, A. Siitonen, M. Vaara, and J. Kirveskari. 2009. New 16-plex PCR method for rapid detection of diarrheagenic Escherichia coli directly from stool samples. Eur. J. Clin. Microbiol. Infect. Dis. 28:899-908.

Benzaquen, M. E., C. A. Risco, L. F. Archbald, P. Melendez, M. J. Thatcher, and W. W. Thatcher. 2007. Rectal temperature, calving-related factors, and the incidence of puerperal metritis in postpartum dairy cows. J. Dairy Sci. 90:2804-2814.

Bicalho, R. C., S. H. Cheong, K. N. Galvao, L. D. Warnick, and C. L. Guard. 2007a. Effect of twin birth calvings on milk production, reproductive performance, and survival of lactating cows. J. Am. Vet. Med. Assoc. 231:1390-1397.

Bicalho, R. C., K. N. Galvao, S. H. Cheong, R. O. Gilbert, L. D. Warnick, and C. L. Guard. 2007b. Effect of stillbirths on dam survival and reproduction performance in Holstein dairy cows. J. Dairy Sci. 90:2797-2803.

de Brito, B. G., L. C. Gaziri, and M. C. Vidotto. 2003. Virulence factors and clonal relationships among Escherichia coli strains isolated from broiler chickens with cellulitis. Infect. Immun. 71:4175-4177.

De Rycke, J., and E. Oswald. 2001. Cytolethal distending toxin (CDT): A bacterial weapon to control host cell proliferation? FEMS Microbiol. Lett. 203:141-148.

Dohmen, M. J., K. Joop, A. Sturk, P. E. Bols, and J. A. Lohuis. 2000. Relationship between intra-uterine bacterial contamination, endotoxin levels and the development of endometritis in postpartum cows with dystocia or retained placenta. Theriogenology 54:1019-1032.

Edmonson, A. J., I. J. Lean, L. D. Weaver, T. Farver, and G. Webster. 1989. A body condition scoring chart for Holstein dairy cows. J. Dairy Sci. 72:68-78.

Fukushima, M., K. Kakinuma, and R. Kawaguchi. 2002. Phylogenetic analysis of Salmonella, Shigella, and Escherichia coli strains on the basis of the gyrB gene sequence. J. Clin. Microbiol. 40:2779 2785 .

Germon, P., Y. H. Chen, L. He, J. E. Blanco, A. Bree, C. Schouler, S. H. Huang, and M. Moulin-Schouleur. 2005. ibeA, a virulence factor of avian pathogenic Escherichia coli. Microbiology 151:1179 1186.

Higgins, J., C. Hohn, S. Hornor, M. Frana, M. Denver, and R. Joerger. 2007. Genotyping of Escherichia coli from environmental and animal samples. J. Microbiol. Methods 70:227-235. 
Huang, S. H., Z. S. Wan, Y. H. Chen, A. Y. Jong, and K. S. Kim. 2001. Further characterization of Escherichia coli brain microvascular endothelial cell invasion gene $i b e A$ by deletion, complementation, and protein expression. J. Infect. Dis. 183:1071-1078.

Johnson, J. R., P. Delavari, M. Kuskowski, and A. L. Stell. 2001. Phylogenetic distribution of extraintestinal virulence-associated traits in Escherichia coli. J. Infect. Dis. 183:78-88.

Johnson, J. R., M. A. Kuskowski, T. T. O’Bryan, and J. N. Maslow. 2002. Epidemiological correlates of virulence genotype and phylogenetic background among Escherichia coli blood isolates from adults with diverse-source bacteremia. J. Infect. Dis. 185:14391447.

Johnson, T. J., Y. Wannemuehler, S. J. Johnson, A. L. Stell, C. Doetkott, J. R. Johnson, K. S. Kim, L. Spanjaard, and L. K. Nolan. 2008. Comparison of extraintestinal pathogenic Escherichia coli strains from human and avian sources reveals a mixed subset representing potential zoonotic pathogens. Appl. Environ. Microbiol. 74:7043-7050.

Kaper, J. B., J. P. Nataro, and H. L. Mobley. 2004. Pathogenic Escherichia coli. Nat. Rev. Microbiol. 2:123-140.

Lally, E. T., R. B. Hill, I. R. Kieba, and J. Korostoff. 1999. The interaction between RTX toxins and target cells. Trends Microbiol. $7: 356-361$.

Langermann, S., S. Palaszynski, M. Barnhart, G. Auguste, J. S. Pinkner, J. Burlein, P. Barren, S. Koenig, S. Leath, C. H. Jones, and S. J. Hultgren. 1997. Prevention of mucosal Escherichia coli infection by Fim $\mathrm{H}$-adhesin-based systemic vaccination. Science 276:607-611.

Linden, T. C., R. C. Bicalho, and D. V. Nydam. 2009. Calf birth weight and its association with calf and cow survivability, disease incidence, reproductive performance, and milk production. J. Dairy Sci. 92:2580-2588.

Malinen, E., A. Kassinen, T. Rinttila, and A. Palva. 2003. Comparison of real-time PCR with SYBR green I or 5'-nuclease assays and dot-blot hybridization with rDNA-targeted oligonucleotide probes in quantification of selected faecal bacteria. Microbiology 149:269-277.

McDougall, S., R. Macaulay, and C. Compton. 2007. Association between endometritis diagnosis using a novel intravaginal device and reproductive performance in dairy cattle. Anim. Reprod. Sci. 99:9-23.

Moreno, E., J. R. Johnson, T. Perez, G. Prats, M. A. Kuskowski, and A. Andreu. 2009. Structure and urovirulence characteristics of the fecal Escherichia coli population among healthy women. Microbes Infect. 11:274-280.

Moreno, E., I. Planells, G. Prats, A. M. Planes, G. Moreno, and A. Andreu. 2005. Comparative study of Escherichia coli virulence determinants in strains causing urinary tract bacteremia versus strains causing pyelonephritis and other sources of bacteremia. Diagn. Microbiol. Infect. Dis. 53:93-99.
Nataro, J. P., T. Steiner, and R. L. Guerrant. 1998. Enteroaggregative Escherichia coli. Emerg. Infect. Dis. 4:251-261.

NRC. 2001. Nutrient Requirements of Dairy Cattle. 7th ed. Natl. Acad. Press, Washington, DC.

Obata-Yasuoka, M., W. Ba-Thein, T. Tsukamoto, H. Yoshikawa, and H. Hayashi. 2002. Vaginal Escherichia coli share common virulence factor profiles, serotypes and phylogeny with other extraintestinal E. coli. Microbiology 148:2745-2752.

Paiva de Sousa, C., and J. D. Dubreuil. 2001. Distribution and expression of the astA gene (EAST1 toxin) in Escherichia coli and Salmonella. Int. J. Med. Microbiol. 291:15-20.

Sheldon, I. M., G. S. Lewis, S. LeBlanc, and R. O. Gilbert. 2006. Defining postpartum uterine disease in cattle. Theriogenology 65:1516-1530.

Sheldon, I. M., A. N. Rycroft, B. Dogan, M. Craven, J. J. Bromfield, A. Chandler, M. H. Roberts, S. B. Price, R. O. Gilbert, and K. W. Simpson. 2010. Specific strains of Escherichia coli are pathogenic for the endometrium of cattle and cause pelvic inflammatory disease in cattle and mice. PLoS ONE 5:e9192.

Silva, E., S. Leitao, T. Tenreiro, C. Pomba, T. Nunes, L. Lopes da Costa, and L. Mateus. 2009. Genomic and phenotypic characterization of Escherichia coli isolates recovered from the uterus of puerperal dairy cows. J. Dairy Sci. 92:6000-6010.

Smith, Y. C., S. B. Rasmussen, K. K. Grande, R. M. Conran, and A. D. O'Brien. 2008. Hemolysin of uropathogenic Escherichia coli evokes extensive shedding of the uroepithelium and hemorrhage in bladder tissue within the first 24 hours after intraurethral inoculation of mice. Infect. Immun. 76:2978-2990.

Struelens, M. J., O. Denis, and H. Rodriguez-Villalobos. 2004. Microbiology of nosocomial infections: Progress and challenges. Microbes Infect. 6:1043-1048.

Tamura, K., and M. Nei. 1993. Estimation of the number of nucleotide substitutions in the control region of mitochondrial DNA in humans and chimpanzees. Mol. Biol. Evol. 10:512-526.

Toth, I., F. Herault, L. Beutin, and E. Oswald. 2003. Production of cytolethal distending toxins by pathogenic Escherichia coli strains isolated from human and animal sources: Establishment of the existence of a new cdt variant (type IV). J. Clin. Microbiol. 41:4285-4291.

Veilleux, S., and J. D. Dubreuil. 2006. Presence of Escherichia coli carrying the EAST1 toxin gene in farm animals. Vet. Res. 37:3-13.

Wang, G., T. S. Whittam, C. M. Berg, and D. E. Berg. 1993. RAPD (arbitrary primer) PCR is more sensitive than multilocus enzyme electrophoresis for distinguishing related bacterial strains. Nucleic Acids Res. 21:5930-5933.

Zerbe, H., C. Ossadnik, W. Leibold, and H. J. Schuberth. 2001. Influence of Escherichia coli and Arcanobacterium pyogenes isolated from bovine puerperal uteri on phenotypic and functional properties of neutrophils. Vet. Microbiol. 79:351-365. 